\title{
The Mass of the $b$ Quark from Lattice NRQCD
}

\author{
S. Collins \\ The Department of Physics and Astronomy, The University of Glasgow, \\ Scotland, G12 8QQ
}

\begin{abstract}
We present results for the mass of the $b$ quark in the $\overline{M S}$ scheme obtained by calculating the binding energy of the $B$ meson in the static limit. The self energy of a static quark, $E_{0}^{\infty}$ needed for this purpose, is now known to $O\left(\alpha^{3}\right)$ in the quenched approximation. We find a preliminary value of $\overline{m_{b}}\left(\overline{m_{b}}\right)=4.34(7) \mathrm{GeV}$ at $n_{f}=0$. The error is dominated by the remaining uncertainty in $E_{0}^{\infty}$. In addition, using $E_{0}^{\infty}$ at $O\left(\alpha^{2}\right)$, we estimate that the quark mass is reduced by approximately $70 \mathrm{MeV}$ when two flavours of dynamical quarks are introduced.
\end{abstract}

\section{Introduction}

The mass of the $b$ quark in the $\overline{M S}$ scheme $\left(\overline{m_{b}}\right)$ can be extracted on the lattice using NRQCD, via the pole mass, $M_{\text {pole }}$, by calculating the binding energy of the $B$ meson, $\bar{\Lambda}_{\text {bind }}: M_{\text {pole }}=M_{B}^{\text {expt }}-\bar{\Lambda}_{\text {bind }}$ where $M_{B}^{\text {expt }}$ is the spin-average of the experimental $B$ and $B^{*}$ masses and $\bar{\Lambda}_{b i n d}=E_{\text {sim }}-E_{0}$. $E_{\text {sim }}$ is the energy of the $B$ meson (at rest) in NRQCD and $E_{0}$ is the $b$ quark self energy. The pole mass is then converted to $\overline{m_{b}}$ at some scale $\mu$ using the continuum perturbative factor $Z_{\text {cont }}: \overline{m_{b}}(\mu)=Z_{\text {cont }}(\mu) M_{\text {pole }}$. While $M_{\text {pole }}$ has an $O\left(\Lambda_{Q C D}\right)$ renormalon ambiguity, this is cancelled by similar effects in $Z_{\text {cont }}: \overline{m_{b}}$ is well defined.

At present, $E_{0}$ is only known to $O(\alpha)$ for the $b$ quark. However, in the limit of the $b$ quark mass becoming infinite, $E_{0}$ is known to $O\left(\alpha^{3}\right)$ if internal quark loops are neglected (the quenched approximation). The tadpole-improved formula can be expressed as

$$
E_{0}^{\infty}=1.070 \alpha_{p}+0.118 \alpha_{p}^{2}-0.3(1.4) \alpha_{p}^{3} \quad: \quad \alpha_{p}=\alpha_{p}^{\left(n_{f}=0\right)}(0.84 / a) .
$$

The $\alpha_{p}^{3}$ coefficient has been determined by Lepage et. al. 1 . The error on the coefficient is numerical and quite large. However, it provides a realistic estimate of the uncertainty in $E_{0}$, compared to using 2 - loop perturbation theory and assuming the contribution of higher order terms is $1 \alpha_{p}^{3}$.

In Eq. 1, $E_{0}$ is expressed in terms of a coupling constant defined on the lattice from the plaquette, $\alpha_{p}$ 2 , evaluated at a characteristic gluon momenta, $q *=0.84 / a$, calculated using the BLM proceedure H. In . Indition, the lattice calculation of $E_{\text {sim }}$ has been tadpole improved, whereby all gauge fields on the lattice are divided by a 'mean-field' approximation to the gluon field, $u_{0}$, 
to obtain more continuum-like operators. The corresponding tadpole improvement of $E_{0}$ leads to the addition of the perturbative series for $\ln u_{0}$. These ingredients result in a well behaved perturbative series for $E_{0}$. This is certainly not the case if the bare lattice coupling, $\alpha_{L}=g_{0}^{2} /(4 \pi)$ is used:

$$
E_{0}^{\infty}=2.1173 \alpha_{L}+11.152 \alpha_{L}^{2}+82.7(1.4) \alpha_{L}^{3}
$$

Di Renzo et. al. 5 have also determined the $O\left(\alpha^{3}\right)$ coefficient. They obtain 86.2(.6), when $\alpha_{L}$ is used. Encouragingly, the two determinations, which have very different systematic errors, agree within $3 \sigma$.

$Z_{\text {cont }}$ has been calculated to $\alpha_{p}^{3}$ by Melnikov and van Ritbergen 6 :

$$
Z_{\text {cont }}=1-0.4244 \alpha_{p}-0.4771 \alpha_{p}^{2}-1.814 \alpha_{p}^{3} \quad: \quad \alpha_{p}=\alpha_{p}^{(0)}\left(0.62 \overline{m_{b}}\right) .
$$

The series is well-behaved and we estimate the uncertainty in $Z_{\text {cont }}$ to be $3 \alpha_{p}^{4}$.

The error introduced by working in the static limit, i.e. ignoring $O\left(\Lambda_{Q C D} / M\right)$ contributions to $\bar{\Lambda}_{\text {bind }}$, leads to $\approx 1 \%$ uncertainty in $\bar{m}_{b}$. The error arising from working in the quenched approximation is also likely to be around 1\% (assuming a $10-20 \%$ shift in $E_{\text {sim }}$ when sea quarks are included). These effects are the same size as the error arising from the numerical error in $E_{0}$.

We obtained $E_{\text {sim }}^{\infty}$ by extrapolating the simulation energy calculated at finite heavy quark mass. The latter was obtained as part of a high statistics study of the $B$ meson spectrum in the quenched approximation at three lattice spacings $(a)$, with $a^{-1}=1-2.5 \mathrm{GeV}$. For details of the simulations see referencel. Note that we use the spin-average of the experimental masses for the $B$ and $B^{*}$ mesons in the expression for $M_{\text {pole }}$ in order to reduce the error in using $E_{\text {sim }}^{\infty}$. In addition, we performed a study of sea quark effects using results obtained from a simulation including two flavours of sea quarks $\left(n_{f}=2\right)$ with $a^{-1} \sim 2 \mathrm{GeV}$. Only the $O\left(\alpha_{p}^{2}\right)$ coefficient for $n_{f}=2$ has been computed $\mathrm{O}$ and hence the comparison with $\overline{m_{b}}$ at $n_{f}=0$ is performed using $E_{0}$ and $Z_{\text {cont }}$ to this order.

\section{Results}

Tables 1 and 2 summarize our results. Within the combined statistical and systematic errors we see that this is the case and we take the result at $\beta=6.0$ as our best determination of $\overline{m_{b}}\left(\overline{m_{b}}\right)=4.34(7) \mathrm{GeV}$ at $n_{f}=0$. Note that the numerical error in $E_{0}$ dominates the uncertainty in $\overline{m_{b}}$.

In addition, using the results at $\beta=6.0$ at $n_{f}=0$ from the $B_{s}$ meson and those obtained at $n_{f}=2$ we see that the $b$ quark mass decreases by $70 \mathrm{MeV}$ at $O\left(\alpha_{p}^{2}\right)$ when sea quarks are introduced. Assuming the systematic (perturbative) errors for the two simulations are correlated this is $\sim 2 \sigma$ in 
Table 1: $\bar{\Lambda}_{\text {bind }}^{\infty}$ and $\overline{m_{b}}\left(\overline{m_{b}}\right)$ in $\mathrm{GeV}$ from the $B$ meson at $n_{f}=0$. The statistical and main systematic errors are estimated, including those due to determining the inverse lattice spacing $\left(a^{-1}\right)$ and residual discretisation effects in $E_{\text {sim }} \sim O\left(\left(\Lambda_{Q C D} a\right)^{2}\right)$.

\begin{tabular}{|c|c|c|c|c|c|c|c|c|c|c|c|}
\hline & \multicolumn{5}{|c|}{$\bar{\Lambda}_{\text {bind }}^{\infty}$} & \multicolumn{6}{|c|}{$\overline{m_{b}}\left(\overline{m_{b}}\right)$} \\
\hline$\beta$ & & stat. & $\begin{array}{c}E_{0}^{\infty} \\
1.4 \alpha_{p}^{3}\end{array}$ & $a^{-1}$ & disc. & & $\begin{array}{c}Z_{\text {cont }} \\
3 \alpha_{p}^{4} \\
\end{array}$ & stat & $\begin{array}{c}E_{0}^{\infty} \\
1.4 \alpha_{p}^{3} \\
\end{array}$ & $a^{-}$ & disc. \\
\hline 5.7 & .24 & (1) & $(11)$ & (1) & (9) & 4.43 & $(3)$ & (1) & $(10)$ & (1) & $(8)$ \\
\hline 6.0 & .35 & $(2)$ & $(6)$ & (1) & (4) & 4.34 & (3) & $(2)$ & $(5)$ & (1) & (3) \\
\hline 6.2 & .36 & (8) & (5) & $(2)$ & (2) & 4.32 & (3) & (7) & (4) & $(2)$ & (2) \\
\hline
\end{tabular}

Table 2: The change in $\overline{m_{b}}\left(\overline{m_{b}}\right)$ from $n_{f}=0$ to 2 , where 2-loop perturbation theory is used.

\begin{tabular}{|ccc|}
\hline$n_{f}$ & $\overline{m_{b}}\left(\overline{m_{b}}\right)$ & stat. error \\
\hline 0 & 4.45 & .01 \\
2 & 4.38 & .02 \\
\hline
\end{tabular}

the (remaining) statistical errors and the same size as the error in $\overline{m_{b}}\left(\overline{m_{b}}\right)$ at $O\left(\alpha_{p}^{3}\right)$. Further work is necessary to reduce the error in $E_{0}$, in order for sea quark and $O\left(\Lambda_{Q C D} / M\right)$ effects to be significant.

\section{Acknowledgements}

The author acknowledges support from the Royal Society of Edinburgh.

\section{References}

1. H. Trottier, private communication and G. P. Lepage et. al., Nucl. Phys. Proc. Suppl. 83-84, 866 (2000).

2. C. T. H. Davies et. al., Phys. Lett. B 345, 42 (1995).

3. G. P. Lepage and P. Mackenzie, Phys. Rev. D 48, 2250 (1993).

4. S. J. Brodsky et. al., Phys. Rev. D 28, 228 (1983).

5. F. Di Renzo, private communication and G. Burgio et. al., Nucl. Phys. Proc. Suppl. 83-84, 935 (2000).

6. K. Melnikov and T. van Ritbergen, Phys.Lett. B482, 99 (2000).

7. J.Hein et. al., Phys. Rev. D 62, 074503 (2000).

8. S. Collins et. al., Phys. Rev. D 60, 074504 (1999).

9. J. Shigemitsu, private communication. 